\title{
Transfer-free Growth of Multilayer Graphene using Self-assembled Monolayers
}

\author{
Gwangseok Yang ${ }^{*}$, Hong-Yeol Kim ${ }^{*}$, Soohwan Jang ${ }^{\S}$ and Jihyun Kim ${ }^{*}$ \\ ${ }^{ \pm}$Department of Chemical and Biological Engineering, Korea University, Seoul 02841, South \\ Korea \\ ${ }^{\S}$ Department of Chemical Engineering, Dankook University, Yongin 16890, South Korea

\section{Corresponding Authors} \\ *E-mail addresses: gunbust99@korea.ac.kr (Hong-Yeol Kim) and hyunhyun7@korea.ac.kr \\ (Jihyun Kim)
}



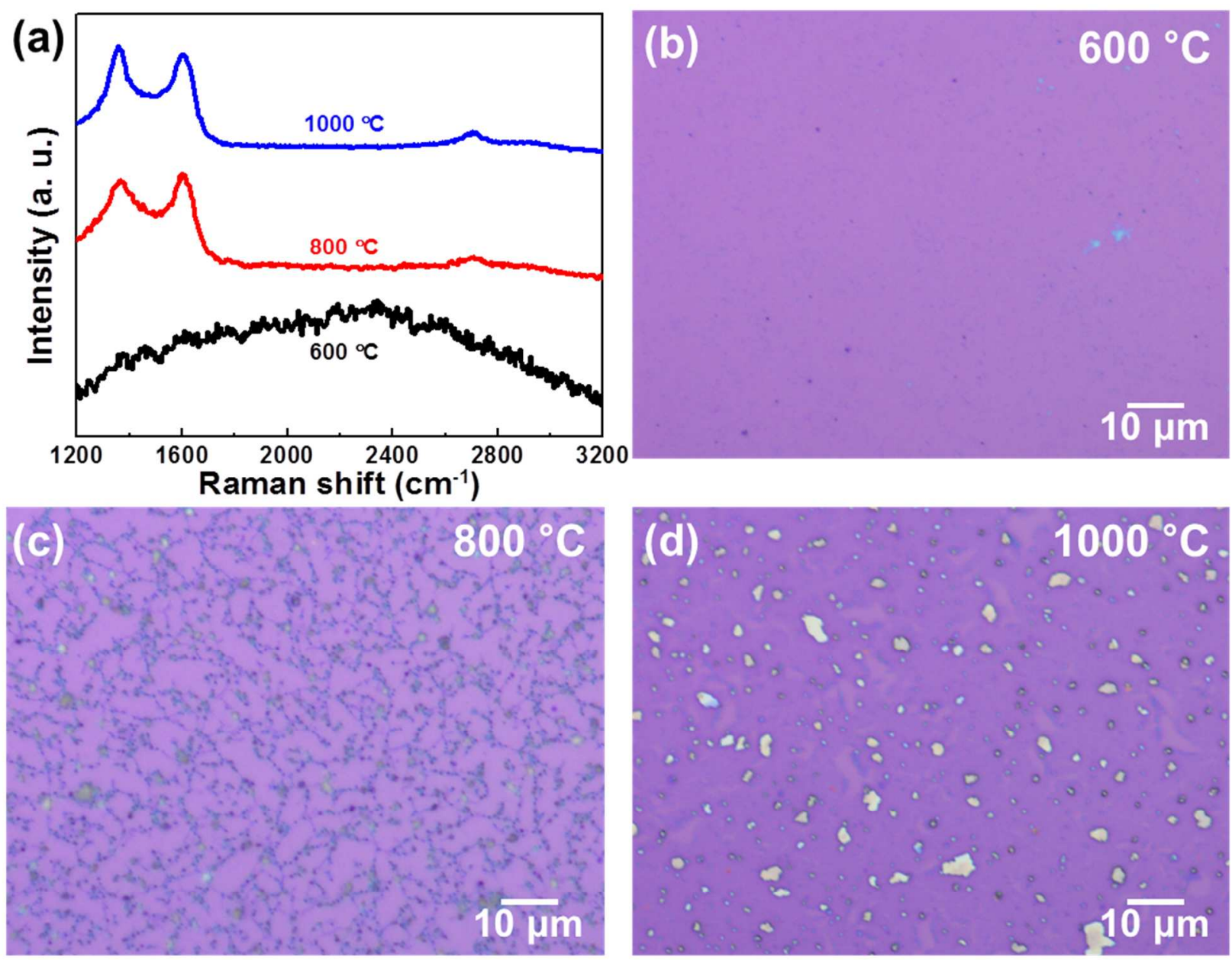

Figure S1. (a) Evolution of Raman spectra of graphene grown using Ni catalyst on $\mathrm{SiO}_{2} / \mathrm{Si}$ substrate with varying growth temperatures. Optical images corresponding to each growth temperature: (b) $600{ }^{\circ} \mathrm{C}$; (c) $800{ }^{\circ} \mathrm{C}$; and (d) $1000^{\circ} \mathrm{C}$. 



Figure S2. Optical images of graphene directly grown on $\mathrm{SiO}_{2} / \mathrm{Si}$ substrate with different $\mathrm{Ar}$ flow rates of: (a) $10 \mathrm{sccm}$; (b) $50 \mathrm{sccm}$; and (c) $500 \mathrm{sccm}$. 

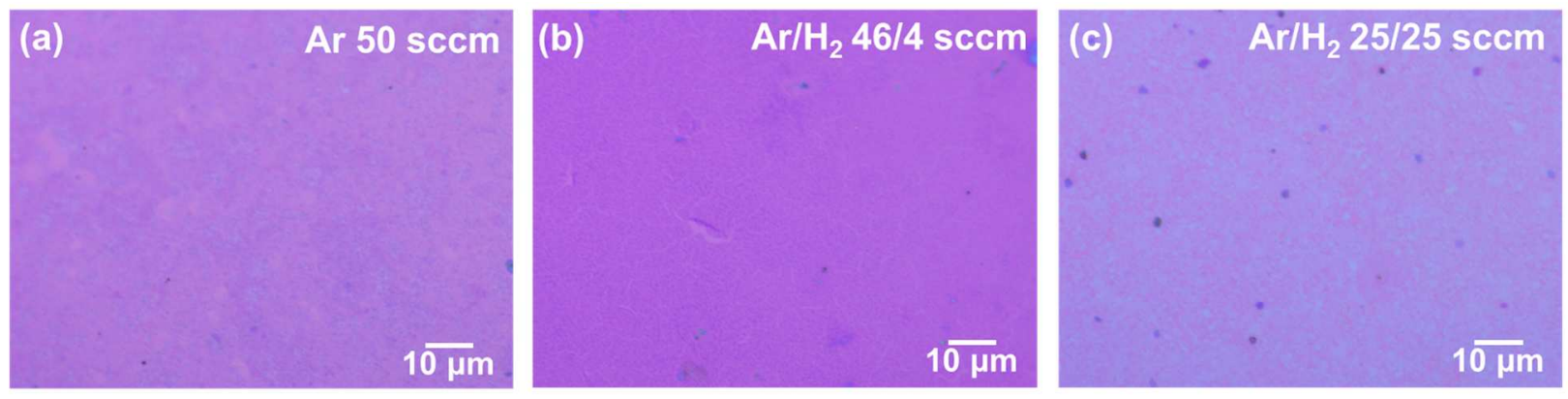

Figure S3. Optical images of graphene directly grown on $\mathrm{SiO}_{2} / \mathrm{Si}$ substrate with different ratios of Ar: $\mathrm{H}_{2}$ flow rates: (a) 50:0; (b) 46:4; and (c) 25:25. 
(a)

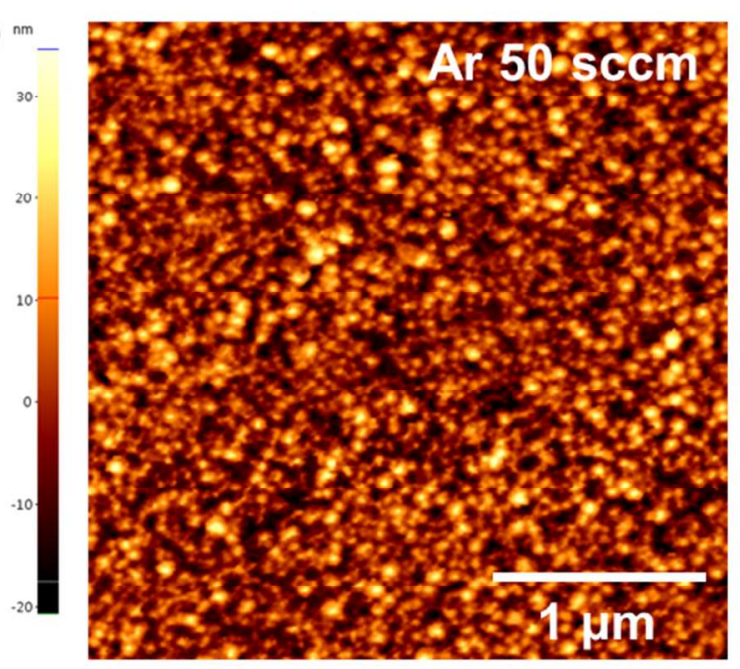

(b)

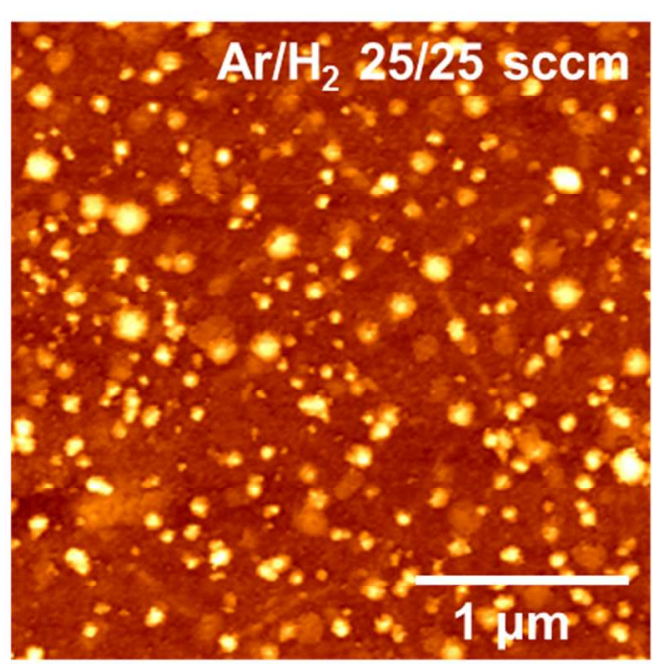

Figure S4. AFM images of the surface of graphene samples produced when the ratio of Ar: $\mathrm{H}_{2}$ flow rate is (c) 50:0 and (d) 25:25. 

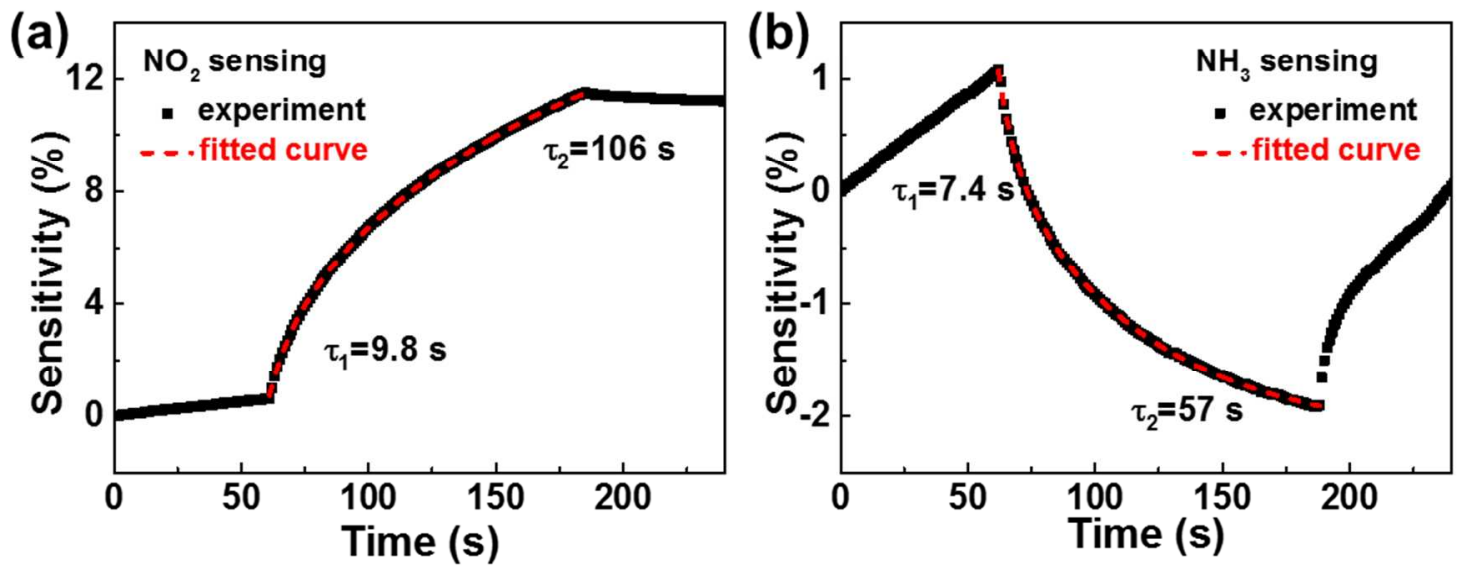

Figure S5. Time-resolved sensitivity and fitted curve of $\mathrm{NO}_{2}$ molecules (a) and $\mathrm{NH}_{3}$ molecules (b), where $\tau_{1}$ and $\tau_{2}$ mean fast and slow response time calculated by bi-exponential function. 

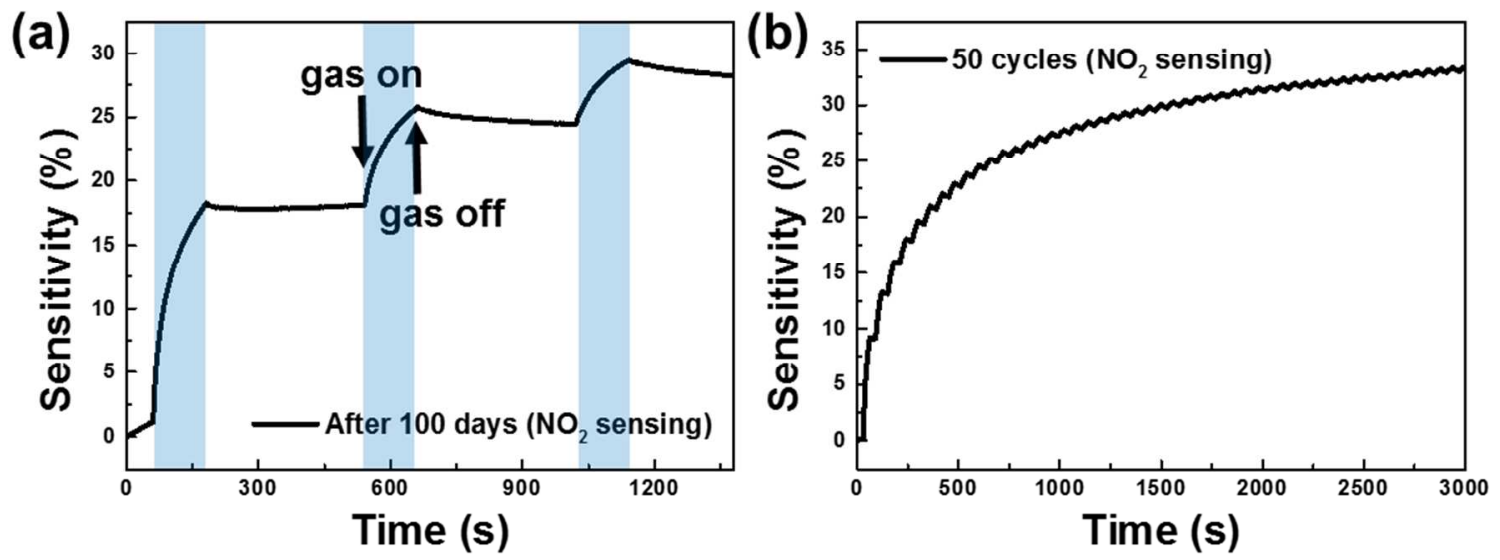

Figure S6. (a) Time-resolved sensitivity towards $\mathrm{NO}_{2}$ gas molecules after 100 days for longterm stability test and (d) time-dependent sensitivity of repeatedly cycled $\mathrm{NO}_{2}$ exposure. 
Table S1. Summary of recently reported graphene-based sensors for $\mathrm{NO}_{2}$ sensing.

\begin{tabular}{|c|c|c|c|c|}
\hline Sensing material & Target gas & sensitivity & Limit of detection & Ref \\
\hline $\begin{array}{l}\text { CVD single-layer } \\
\text { graphene }\end{array}$ & $\mathrm{NO}_{2}$ & $\begin{array}{c}32 \% / 200 \mathrm{ppm} \\
\left(\Delta \mathrm{R} / \mathrm{R}_{0}\right)\end{array}$ & - & (1) \\
\hline $\begin{array}{l}\text { CVD single-layer } \\
\text { graphene }\end{array}$ & $\mathrm{NO}_{2}$ & $\begin{array}{c}40 \% / 200 \mathrm{ppm} \\
\left(\Delta \mathrm{R} / \mathrm{R}_{0}\right)\end{array}$ & - & (2) \\
\hline CVD graphene & $\mathrm{NO}_{2}$ & $\begin{array}{c}51 \% / 40 \mathrm{ppm} \\
\left(\Delta \mathrm{R} / \mathrm{R}_{0}\right)\end{array}$ & - & (3) \\
\hline CVD graphene & $\mathrm{NO}_{2}$ & $\begin{array}{c}4 \% / 100 \mathrm{ppb} \\
\left(\Delta \mathrm{R} / \mathrm{R}_{0} \text { (sheet }\right. \\
\text { resistance change)) }\end{array}$ & $100 \mathrm{ppb}$ & (4) \\
\hline $\begin{array}{c}\text { epitaxially grown } \\
\text { graphene on } 6 \mathrm{H}-\mathrm{SiC}\end{array}$ & $\mathrm{NO}_{2}$ & $\begin{array}{c}2.25 \% / 500 \mathrm{ppb} \\
\left(\Delta \mathrm{G} / \mathrm{G}_{0}\right)\end{array}$ & - & (5) \\
\hline 3D RGO hydrogel & $\mathrm{NO}_{2}$ & $\begin{array}{c}0.19 \% / 2 \mathrm{ppm} \\
\left(\Delta \mathrm{R} / \mathrm{R}_{0}\right)\end{array}$ & $187 \mathrm{ppb}$ & (6) \\
\hline $\begin{array}{c}\text { Ozone-treated CVD } \\
\text { graphene }\end{array}$ & $\mathrm{NO}_{2}$ & $\begin{array}{c}19.7 \% / 200 \mathrm{ppm} \\
\left(\Delta \mathrm{R} / \mathrm{R}_{0}\right)\end{array}$ & $1.3 \mathrm{ppb}$ & (7) \\
\hline $\begin{array}{c}\mathrm{SnO}_{2}-\mathrm{ZnO} \text { core-shell } \\
\text { nanofiber sensor }\end{array}$ & $\mathrm{NO}_{2}$ & $40 \% / 5 \mathrm{ppm}$ & - & (8) \\
\hline $\mathrm{SnO}_{2}$ thin films & $\mathrm{NO}_{2}$ & $1570 \% / 30$ ppm & - & (9) \\
\hline $\begin{array}{l}\text { Transfer-free } \\
\text { graphene }\end{array}$ & $\mathrm{NO}_{2}$ & $\begin{array}{c}11.5 \% / 200 \mathrm{ppm} \\
\left(\Delta \mathrm{R} / \mathrm{R}_{0}\right)\end{array}$ & - & This work \\
\hline
\end{tabular}




\section{Reference}

(1) Yang, G.; Lee, C.; Kim, J.; Ren, F.; Pearton, S. J., Flexible Graphene-Based Chemical Sensors on Paper Substrates. Phys. Chem. Chem. Phys. 2013, 15, 1798-1801.

(2) Lee, G.; Yang, G.; Cho, A.; Han, J. W.; Kim, J., Defect-Engineered Graphene Chemical Sensors with Ultrahigh Sensitivity. Phys. Chem. Chem. Phys. 2016, 18, 14198-14204.

(3) Choi, H.; Choi, J. S.; Kim, J.-S.; Choe, J.-H.; Chung, K. H.; Shin, J.-W.; Kim, J. T.; Youn, D.-H.; Kim, K.-C.; Lee, J.-I.; Choi, S.-Y.; Kim, P.; Choi, C.-G.; Yu, Y.-J., Flexible Electronics: Flexible and Transparent Gas Molecule Sensor Integrated with Sensing and Heating Graphene Layers (Small 18/2014). Small 2014, 10, 3812-3812.

(4) Yavari, F.; Castillo, E.; Gullapalli, H.; Ajayan, P. M.; Koratkar, N., High Sensitivity Detection of No2 and Nh3 in Air Using Chemical Vapor Deposition Grown Graphene. Appl. Phys. Lett. 2012, 100, 203120.

(5) Nomani, M. W. K.; Shishir, R.; Qazi, M.; Diwan, D.; Shields, V. B.; Spencer, M. G.; Tompa, G. S.; Sbrockey, N. M.; Koley, G., Highly Sensitive and Selective Detection of No2 Using Epitaxial Graphene on 6h-Sic. Sens. Actuators B-Chem. 2010, 150, 301-307.

(6) Wu, J.; Tao, K.; Miao, J.; Norford, L. K., Improved Selectivity and Sensitivity of Gas Sensing Using a 3d Reduced Graphene Oxide Hydrogel with an Integrated Microheater. Acs Appl. Mater. Mater. Interfaces 2015, 7, 27502-27510.

(7) Chung, M. G.; Kim, D. H.; Lee, H. M.; Kim, T.; Choi, J. H.; Seo, D. k.; Yoo, J.-B.; Hong, S.H.; Kang, T. J.; Kim, Y. H., Highly Sensitive No2 Gas Sensor Based on Ozone Treated Graphene. Sens. Actuators B-Chem. 2012, 166-167, 172-176.

(8) Sun-Woo, C.; Jae Young, P.; Sang Sub, K., Synthesis of Sno 2 -Zno Core-Shell Nanofibers Via a Novel Two-Step Process and Their Gas Sensing Properties. Nanotechnology 2009, 20, 465603.

(9) Kunimoto, A.; Abe, N.; Uchida, H.; Katsube, T., Highly Sensitive Semiconductor Nox Gas Sensor Operating at Room Temperature. Sens. Actuators B-Chem. 2000, 65, 122-124. 